OPEN ACCESS

Edited by:

Robert Braidwood Sim University of Oxford, United Kingdom

Reviewed by: Miki Nakao,

Kyushu University, Japan Kenneth Reid,

University of Oxford, United Kingdom

${ }^{*}$ Correspondence: Sivan Livson sivan.livson@hus.fi

Seppo Meri

Seppo.meri@helsinki.fi

Specialty section: This article was submitted to Molecular Innate Immunity, a section of the journal

Frontiers in Immunology

Received: 17 May 2020 Accepted: 24 November 2020

Published: 11 January 2021

Citation: Livson S, Jarva H, Kalliala I, Lokki Al, Heikkinen-Eloranta J, Nieminen P and

Meri S (2021) Activation of the

Complement System in the Lower Genital Tract During Pregnancy and Delivery. Front. Immunol. 11:563073. doi: 10.3389/fimmu.2020.563073

\section{Activation of the Complement System in the Lower Genital Tract During Pregnancy and Delivery}

\author{
Sivan Livson ${ }^{1,2 *}$, Hanna Jarva ${ }^{2,3}$, Ilkka Kalliala ${ }^{1,4,5}$, A. Inkeri Lokki ${ }^{1,2}$, \\ Jenni Heikkinen-Eloranta ${ }^{1}$, Pekka Nieminen ${ }^{1,6}$ and Seppo Meri ${ }^{2,3^{*}}$ \\ ${ }^{1}$ Department of Obstetrics and Gynecology, Helsinki University Central Hospital, University of Helsinki, Helsinki, Finland, \\ ${ }^{2}$ Department of Bacteriology and Immunology and Translational Immunology Research Program, University of Helsinki, \\ Helsinki, Finland, ${ }^{3}$ HUS Diagnostic Center, Helsinki University Hospital Laboratory, Helsinki University Hospital, Helsinki, \\ Finland, ${ }^{4}$ Department of Surgery and Cancer, Institute of Reproductive and Developmental Biology, Imperial College London, \\ London, United Kingdom, ${ }^{5}$ Department of Metabolism, Digestion and Reproduction, Faculty of Medicine, Imperial College \\ London, London, United Kingdom, ${ }^{6}$ Human Microbiome Research Program, Faculty of Medicine, University of Helsinki, \\ Helsinki, Finland
}

Background: Human pregnancy alters profoundly the immune system. The local involvement and mechanisms of activation of the complement system in the cervicovaginal milieu during pregnancy and delivery remain unexplored.

Objectives: To determine whether normal pregnancy and delivery are associated with local activation of complement or changes in the immunoglobulin profile in the cervix.

Study Design: This study was designed to assess IgA, IgG, and complement activation in the cervicovaginal area in three groups of patients: i) 49 pregnant women (week 41+3$42+0)$ not in active labor, ii) 24 women in active labor (38+4-42+2), and iii) a control group of nonpregnant women $(n=23)$ at child-bearing age. We collected mucosal samples from the lateral fornix of the vagina and external cervix during routine visits and delivery. The Western blot technique was used to detect complement C3 and its activation products. For semiquantitative analysis, the bands of the electrophoresed proteins in gels were digitized on a flatbed photo scanner and analyzed. IgA and IgG were analyzed by Western blotting and quantified by ELISA. One-way ANOVA and Tukey's Multiple Comparison tests were used for statistical comparisons.

Results: A higher abundance but lower activation level of C3 in both the external cervix $(P<0.001)$ and lateral fornix of the vagina $(P<0.001)$ was observed during delivery $(58 \pm 22$, $\mathrm{n}=24)$ in comparison to the groups of nonpregnant (72 $\pm 13 \%$; mean $\pm S D, n=23)$ and pregnant women $(78 \pm 22 \%, n=49)$. Complement activating lgG was detected in higher abundance than $\lg \mathrm{A}$ in the cervicovaginal secretions of pregnant women. In a small proportion samples also C3-lgG complexes were detected. 
Conclusions: Our results reveal an unexpectedly strong activation of the complement system and the presence lgG immunoglobulins in the cervicovaginal area during pregnancy, active labor, and among nonpregnant women. In contrast to the higher amounts of C3 in the cervicovaginal secretions during labor, its activation level was lower. Complement activating IgG was detected in higher concentrations than IgA in the mucosal secretions during pregnancy and labor. Taken together our results imply the presence a locally operating humoral immune system in the cervicovaginal mucosa.

Keywords: uterine cervix, vaginal mucosa, IgG, IgA, C3, parturition, inflammation, delivery

\section{INTRODUCTION}

A unique phenomenon of successful coexistence of the maternal immune system and the semi-allograft fetoplacental unit is seen in pregnant women (1-3). Growing knowledge about the immunological microenvironment within the female genital tract has increased the interest in understanding the immunological processes and their role in parturition (4-6). Reports describing chemotactic recruitment and activation of inflammatory neutrophils and macrophages into the uterus, decidua, fetal membranes and cervix during labor point towards local rather than systemic inflammatory events $(7-10)$. The innate immune system has been linked to these processes and to the generation of a sterile pro-inflammatory state that will pave the way to labor and delivery of the baby $(11,12)$.

The complement (C) system is part of innate immunity (13, 14). The proteolytic activation cascades of $C$ comprise about 50 proteins (15). Complement can become activated through three distinct pathways: the classical, lectin and alternative pathways (16). These pathways each converge at the central step of the $\mathrm{C}$ system, i.e. the cleavage of C3 by C3 convertases (16). Complement functions in antimicrobial defense and as an opsonophagocytic clean-up system of the body together with phagocytes. Activation products generated include e.g. the anaphylatoxins $\mathrm{C} 3 \mathrm{a}$ and $\mathrm{C} 5 \mathrm{a}$ that can induce major physiological changes, like contracting smooth muscle and increasing vascular permeability (17). Pregnancy and parturition are associated with increased levels of $\mathrm{C}$ components and with $\mathrm{C}$ activation in the blood (18-26). The presence of $\mathrm{C}$ components in tissues is a result of their diffusion from blood plasma and local production by different cell types including macrophages, fibroblasts, and endothelial cells. Information about the local role of $\mathrm{C}$ in the cervicovaginal area in humans is scarce.

Under normal circumstances $\mathrm{C}$ activation is well regulated and only minimal deposition of its activated components, including $\mathrm{C} 1 \mathrm{q}, \mathrm{C} 4 \mathrm{~b}, \mathrm{C} 3 \mathrm{~b} / \mathrm{iC} 3 \mathrm{~b}$, or the membrane attack complex (MAC), occurs in the mucosa. However, in a variety of adverse pregnancy outcomes dysregulation of $\mathrm{C}$ has been demonstrated. These include hypertensive diseases of pregnancy $(27,28)$, antiphospholipid antibody syndrome-associated fetal loss (29), recurrent miscarriage and preterm birth (30-33). To our knowledge, local activation of the $\mathrm{C}$ system in the cervicovaginal mucosa in humans and its relation to timing of parturition have not yet been examined.
We hypothesized that, because of e.g. a strong microbial exposure in the lower genital tract, the $\mathrm{C}$ system is constantly active, but should be tightly regulated at the time of parturition in order to protect the mother and fetus from an immune attack. Our present study aimed at investigating whether and to what extent $\mathrm{C}$ is activated in the cervicovaginal area, and whether local $\mathrm{C} 3$ activation is related to the parturition process.

\section{MATERIALS AND METHODS}

\section{Study Subjects and Samples}

To address the role of complement in parturition, a cohort of samples was collected to determine $\mathrm{C}$ activation in the cervicovaginal area. We recruited three groups of study subjects: i) pregnant women $(n=49)$ with pregnancy duration of $41+3$ to 42 +0 weeks in whom labor had not yet become initiated, ii) women in active labor ( $\mathrm{n}=24)(38+4-42+2$ weeks), and iii) non-pregnant women $(n=23)$ in child-bearing age, who had arrived for routine out-patient visits in the clinic. All pregnancies were singleton pregnancies with intact fetal membranes at the time of sampling. None of the women had been previously treated for cervical precancerous lesion. All women were generally healthy with no chronic disease or diagnosed immune deficiency, except for one with IgA deficiency in the pregnant women group. None of the study subjects had used any type of corticosteroids for at least 6 months before sampling. None of the women recruited had had unprotected sexual intercourse for at least $48 \mathrm{~h}$ before sampling. All pregnant individuals were screened for gestational diabetes in mid-pregnancy. Altogether $17 \%$ tested positive. They were treated conservatively and monitored at the maternity clinic with no need for antidiabetic drugs. Demographic and clinical characteristics of the pregnant study subjects are presented in Table $\mathbf{1}$.

All subjects $(n=96)$ were in child-bearing age (17-40 years old). The median BMI value before pregnancy was $23 \pm 2.4$ (median \pm range, $n=73$ ). In the pregnancy, delivery and control groups, systemic or local vaginal usage of antibiotics less than 6 months from sampling was recorded in $22 \%, 21 \%$, and $20 \%$ of the women, respectively (Table 1). In the pregnancy group 53\% $(26 / 49)$ and the labor group 33\% (8/24) of the women were primiparas $(\mathrm{p}<0.05)$.

All samples were collected at the Helsinki University Hospital between October 2015 and March 2017. The study was approved by the Helsinki University Hospital's Ethical Committee (91/13/03/03/ 
TABLE 1 | Patient characteristics.

\begin{tabular}{|c|c|c|c|}
\hline & Non pregnant & Pregnant patients & Patients in labor \\
\hline & $n=23$ & $n=49$ & $n=24$ \\
\hline Age (mean, SD) & $30 \pm 5$ & $32.3 \pm 4.3$ & $30.2 \pm 5$ \\
\hline First delivery (\%) & & 53 & 33 \\
\hline Median prepregnancy BMI & & 23 & 22 \\
\hline Smoking (\%) & 30 & 4.1 & 4.2 \\
\hline Antibiotic consumption in the past 6 months (\%) & & 22 & 21 \\
\hline Pregnancy length in days (median) & & 294 & 282 \\
\hline Range of pregnancy length (days) & & $289-296$ & $270-92$ \\
\hline Average baby weight (g) & & 3700 & 3574 \\
\hline Positive for vaginal group B streptococcus (\%) & & 33 & 35 \\
\hline Artificially induced delivery (\%) & & 61 & 0 \\
\hline Gestational diabetes (\%) & & 15 & 18 \\
\hline
\end{tabular}

2015). All participating women provided written informed consents and were requested to fill up an exploratory questionnaire. Serial samples were collected by two experienced physicians from the lateral fornix of the vagina (LF) and external cervix (EC) using the Rovers Viba-Brush tool (Rovers Medical Devices, Oss, The Netherlands). Ten subjects provided two additional swabs from the EC and LF directly into $10 \mathrm{mM}$ EDTA to control for possible ex vivo $\mathrm{C}$ activation. The additional group of 23 non-pregnant subjects were sampled from the LF. These samples were taken at a colposcopy clinic. Many of the patients came because they may have cervical changes. Thus, samples from the external cervix would not have been representative. All samples were inserted immediately into Eppendorf tubes containing $20 \mu \mathrm{l}$ phosphate-buffered saline, $\mathrm{pH} 7.4$ (PBS) and were frozen into $-80^{\circ} \mathrm{C}$ within $30 \mathrm{~min}$ of sampling. No blood-contaminated samples were included in the study.

\section{Analysis of $\lg G$ and $\lg A$}

IgG and IgA in cervicovaginal samples were analyzed by immunoblotting and quantified by an ELISA assay. Twenty $\mu$ l portions of appropriately diluted samples were loaded onto 4\%$12 \%$ SDS-PAGE gels under reducing conditions. After transferring the proteins to a nitrocellulose filter, nonspecific binding sites were blocked by incubation with 5\% milk in phosphate-buffered saline (PBS) containing 0.05\% Tween 20 detergent. The membranes were then incubated for $1 \mathrm{~h}$ at RT with HRP-conjugated rabbit-anti-human IgG or IgA antibody (Dako; final dilutions 1:10,000 and 1:5,000, respectively, in milk/ $\mathrm{PBS} /$ Tween). Protein bands were visualized by an in-house protocol for electrochemiluminescence. After washing with PBS+0.05\% Tween 20 an enhanced chemiluminescence solution (WesternBright ECL, Advansta, San Jose, CA) that includes hydrogen peroxide was added and films developed at different exposure times.

To quantify levels of IgG and IgA in the cervicovaginal mucosal samples standardized ELISA assays (Bethyl Laboratories, Inc. USA, Catalog no. E101-104 and Catalog no. E100-102, respectively) were used. Human sera with known IgG and IgA concentrations were used as controls. In this assay the intra- and inter-assay coefficients of variations were less than $5 \%$. Mucosal samples and sera were diluted 1:1000, 1:3000, and
$1: 10000$ for $\operatorname{IgG}$ and $1: 10,1: 30,1: 100$, and 1:300 for $\operatorname{IgA}$ and analyzed in duplicate. After incubating the samples for $2 \mathrm{~h}$ on the plate wells they were washed with a buffer (TBS) containing $0.05 \%$ Tween 20 using an automated plate washer (EL $x 50$ Washer, BioTek). Thereafter, HRP-anti human IgG and HRPanti-human IgA (both from Dako), diluted 1:5000 and 1:2000 respectively in PBS were added and incubated for another $2 \mathrm{~h}$ at $22^{\circ} \mathrm{C}$. After washing with TBS, $0.05 \%$ Tween 20 , substrate (OPD) was added. The reaction was stopped with $0.5 \mathrm{M} \mathrm{H}_{2} \mathrm{SO}_{4}$ and a microplate spectrophotometer (SpectraMax, Bio-strategy) was used to measure the optical density of samples at a wave-length of $492 \mathrm{~mm}$.

\section{Analysis of C3 Cleavage in Vaginal Lateral Fornix and External Cervix Samples}

Western blot analyses were performed using in-house protocols (34). The samples were thawn on ice, centrifuged $12,000 \times g$ for 3 min and diluted 1:10 in sterile PBS. $20 \mu$ portions of a dilution series of samples (in PBS) in SDS and 5\% mercaptoethanol containing sample buffer were loaded onto $4 \%-12 \%$ SDS-PAGE gels and run under reducing conditions. A normal human serum (NHS) pool was obtained from healthy laboratory personnel after a written informed consent and used as a reference. The proteins from the gel were electrotransferred to a nitrocellulose filter. To prevent nonspecific binding the nitrocellulose membranes were incubated in 5\% milk in PBS/Tween $0.05 \%$ for $1 \mathrm{~h}$. The membranes were then incubated with rabbit antihuman C3c antibody (Dako; final dilution: 1:10,000 in milk/PBS/ Tween) overnight at $+4^{\circ} \mathrm{C}$. For additional detection of C3-IgG complexes, also rabbit antibodies against C3d (Dako) were used similarly as anti-C3c antibodies. The membranes were washed with PBS/Tween and incubated for $1 \mathrm{~h}$ at RT with HRP-goatanti-rabbit IgG antibody (Jackson ImmunoResearch; 1:10,000 in milk/PBS/Tween). Finally, the membranes were washed, and protein bands were visualized by electrochemiluminescence.

\section{Quantification of C3 Activation}

For quantitative determination of the $\mathrm{C} 3$ bands the films were digitized on a flatbed photo scanner and quantified using ImageJ/ Fiji win-64 software. Activation of C3 results in several split 
products (Figure 2), including C3b, iC3b, C3c, C3dg, and C3d. The level of $\mathrm{C} 3$ activation was determined by assessment of the relative level of intensity of the C3 $\alpha$-chain split products ( $\alpha$-chain fragments in the split products) from total C3 reactivity. The respective $\alpha^{\prime}$-chains of these split products can be visualized by Western blotting and may be quantified in relation to the amount of total C3 $\alpha / \alpha^{\prime}$-chain reactivity (native plus activated C3) found in the same specimen. The $\beta$ chain is not cleaved, thus its amount remains the same regardless of the level of activation. Calculation of the total percentage of $\mathrm{C} 3$ activation was done by calculating the intensity of C3 $\alpha$ ' split products [x 100\%] and dividing the result by [intensity of native $\mathrm{C} 3 \alpha+\mathrm{C} 3 \alpha^{\prime}$ split products].

Results are shown as mean \pm SD values. For comparing the significances of differences between the study groups the twotailed Student's t-test or one-way ANOVA with Tukey's multiple comparison test were used.

\section{RESULTS}

\section{Immunoglobulins IgA and IgG in the Cervicovaginal Samples}

Since IgG is known to activate complement, but IgA is not, we wanted to analyze the relative proportions of these immunoglobulins in the cervical secretions. Figures 1A, B show two representative Western blots for analysis of the IgG and IgA heavy chains, $\gamma$ and $\alpha$, respectively. In the majority of samples $(n=96)$ the IgA heavy chains were intact. For IgG, however, in approximately half of the samples, especially in those taken during delivery, additional bands with a lower molecular weight than that for the intact $50 \mathrm{kDa}$ heavy chain were observed in the blots. This suggests proteolytic cleavage. The intensities of the IgG heavy chains were stronger than those for IgA suggesting that IgG is abundantly present in all the mucosal samples. In one case an apparent IgA deficiency was detected (Figure 1B).

More accurate quantification of IgG and IgA was done by ELISA (Figures 1C, D). On the average, higher concentrations of IgG than IgA were observed in samples from pregnant women (both in those in late pregnancy or in labor; $\mathrm{P}<0.001$ : one-way ANOVA and Tukey's multiple comparison test). In contrast, in nonpregnant women the IgA levels $(1.0 \pm 0.4, \mathrm{n}=19$; mean $\pm \mathrm{SD})$ were higher than IgG levels $(0.5 \pm 0.3, \mathrm{n}=19, \mathrm{P}<0.001$, Student's t-test $)$. The IgG levels were higher in samples taken during labor (EC: $1.7 \pm 0.7, n=22$; LF: $1.6 \pm 0.9, \mathrm{n}=21$ ) or late pregnancy (EC: $1.2 \pm 0.6 \mathrm{mg} / \mathrm{ml}, \mathrm{n}=46$; LF: $1.3 \pm 0.7, \mathrm{n}=30)$ than in those from nonpregnant women $(0.5 \pm 0.3$, $\mathrm{n}=19, \mathrm{P}<0.01$; Tukey's multiple comparison test). Values during labor (both in EC and LF) were slightly higher than in late pregnancy $(\mathrm{P}<0.01)$. No significant difference was detected between the study groups in IgA concentrations.
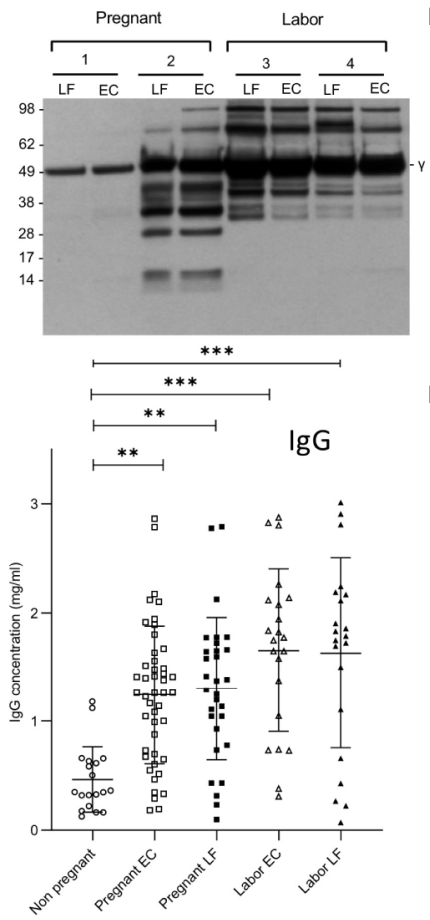

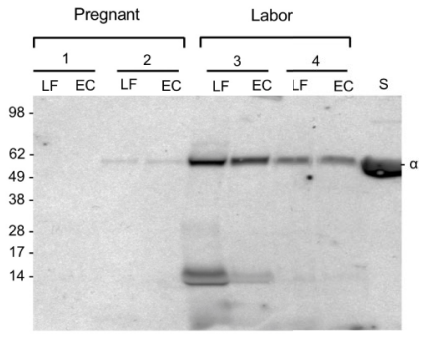

D

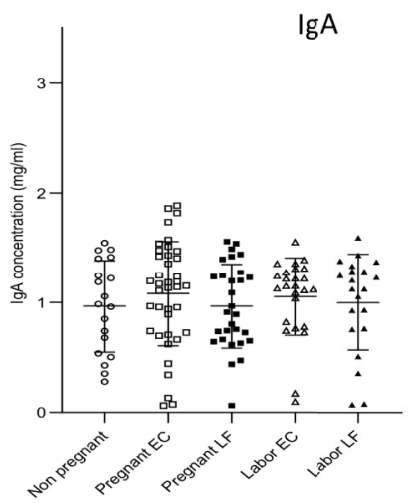

FIGURE 1 | Immunoblotting analysis of IgG and IgA heavy chains and levels of IgG and IgA in EC and LF during pregnancy and labor. Samples 1 and 2 were from pregnant women and samples 3 and 4 from women in labor. As shown in (A), IgG is abundant in all samples. More quantitative variation exists for lgA (B). In the selected samples from pregnant women patient number 1 was found to be lgA-deficient. Antibodies used were specific for the $\gamma$ (IgG) and $\alpha$ (IgA) heavy chains, both approximately $50 \mathrm{kDa}$ in size. Quantification of $\operatorname{lgG}$ (C) and IgA (D) was done by ELISA. Higher concentrations of IgG were found in the EC and LF during pregnancy and labor in comparison to IgA. IgG concentration is significantly higher during pregnancy and during labor both in the EC and the LF in comparison to the nonpregnant controls ( ${ }^{\star \star} \mathrm{P}<0.01,{ }^{\star \star \star} \mathrm{P}<0.001$; one-way ANOVA and Tukey's multiple comparison test). 


\section{Local C3 Activation in Non-Pregnant and Pregnant Women and During Labor}

Our next aim was to study the presence and activation of C3 locally in the cervicovaginal area. Native complement C3 consists of one $\alpha$ and one $\beta$ chain linked together via a disulfide bond. The activation of $\mathrm{C} 3$ is schematically described in Figure 2. The band identities in the immunoblots were determined by comparison to C3 activation fragments in zymosan-activated serum and by inactivating purified $\mathrm{C} 3 \mathrm{~b}$ with factors $\mathrm{H}$ and $\mathrm{I}$ (not shown). During activation, the C3 $\beta$-chain remains intact in the cleavages and thus reflects the total original amount of $\mathrm{C} 3$ in the sample. In addition to the $\alpha$-chain the Western blots traced the $\alpha$ 'chain and its cleavage fragments, which were quantified by using the "ImageJ/Fiji win-64 software J". As shown in Figure 3, C3 in the cervicovaginal samples becomes extensively activated and degraded into multiple cleavage fragments. Occasionally, in the blots a band above the $\mathrm{C} 3$ beta-chain was observed (Figure 3). It is likely an autolytic cleavage product of the C3 alpha-chain, and thus not an activation product.

The average $\mathrm{C} 3$ activation level was $72 \pm 13 \%$ (mean \pm SD, $n=23$ ) in the non-pregnant control group, $78 \pm 22 \%(n=49)$ in the pregnant group and $58 \pm 22 \%(n=24)$ during active labor (Figure 4).
Differences were significant between the pregnant group and the labor group in EC samples $(\mathrm{P}<0.001)$, and between the nonpregnant group and the labor group $(\mathrm{p}<0.01)$ (Figure 4). The results indicate that $\mathrm{C} 3$ in this local environment is in a continuous state of activation. In ten additional swabs immersed directly into $10 \mathrm{mM}$ EDTA for control of possible ex vivo C activation the percentages of cleaved $\mathrm{C} 3$ in the PBS/EDTAcontaining buffer were $62 \pm 23 \%$ (mean \pm SD), and $70 \pm 18 \%$ in the EC and LF samples, respectively. In the non-EDTA containing samples the respective percentages of $\mathrm{C} 3$ activation were $62 \pm 21 \% \mathrm{~s}$ and $87 \pm 22 \%$ in the EC and LF samples. This indicated that, unlike in EC samples, in the LF samples, some $\mathrm{C}$ activation continued still ex vivo.

\section{C3 Activation in Lateral Fornix Compared to External Cervix}

In LF and EC samples from pregnant women and women in labor large amounts of $\mathrm{C} 3$ activation fragments indicated strong local activation of C3. A higher level of C3 activation was observed during pregnancy in LF: $79 \pm 20 \%(n=49)$ in comparison to EC: $57 \pm 30 \%$ $(\mathrm{n}=49)(\mathrm{P}<0.001)$ (Figure 4). No significant difference was seen in values in samples from different locations during labor.
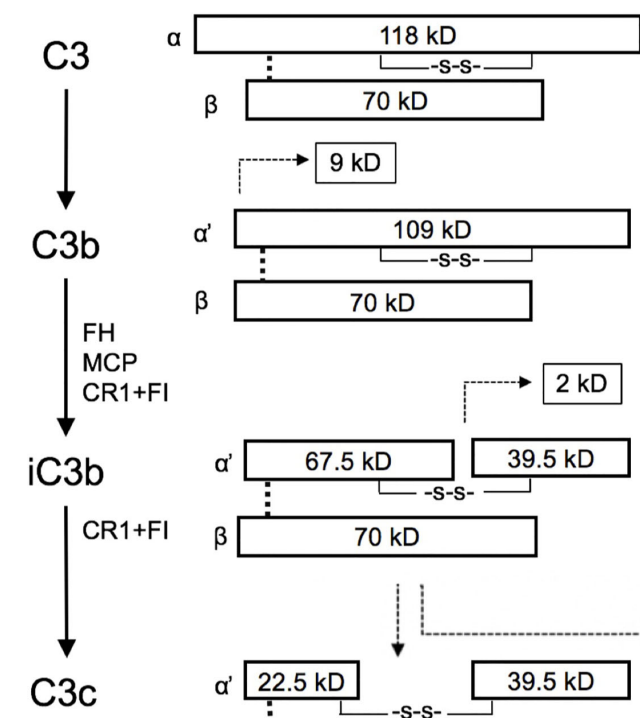

$\beta$
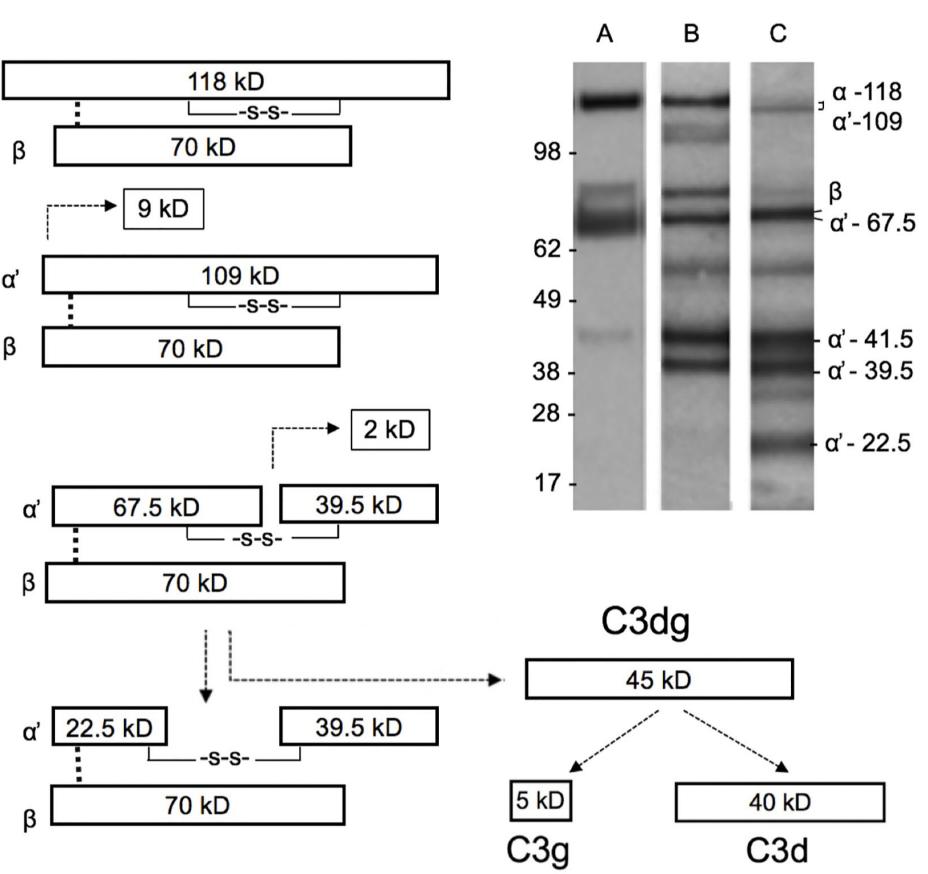

FIGURE 2 | Schematic structure of C3 and its cleavage. The three distinct complement pathways will converge at C3 and promote activation of C3 to C3a and C3b by C3 convertases. The 109 kDa alpha'-chain in C3b becomes cleaved by factor I and a cofactor, e.g. factor H, to smaller 67.5 and $39.5 \mathrm{kDa}$ fragments that are then part of iC3b. iC3b will subsequently get further cleaved to C3c and C3dg. The $\beta$ chain remains uncleaved as a $70 \mathrm{kDa}$ band. Examples of the C3 cleavage patterns (determined by rabbit anti-human $\mathrm{C} 3 \mathrm{C}$ antibody) in our samples are shown in the upper right corner. Lanes $\mathrm{A}$, $\mathrm{B}$, and $\mathrm{C}$ show representative samples of different levels of $\mathrm{C} 3$ breakdown in the patient samples. Lanes $A, B$, and $C$ represent samples containing $C 3 b(A)$, iC3b (B), and a mixture of iC3b and C3c (C), respectively. In lane $\mathrm{B}$, and faintly in lane $\mathrm{C}$, the band at $118-109 \mathrm{kDa}$ represents alpha/alpha-chains, which have remained uncleaved. The identity of the $\approx 55 \mathrm{kDa}$ band is not known, but could represent a proteolytic cleavage fragment of the $67.5 \mathrm{kDa}$ band. Such a fragment could be generated e.g. by plasmin, which is likely present in the mucosal fluids. The fragment above the $70 \mathrm{kDa}$ beta-band could represent an autolytic cleavage product of the alpha-chain, where C3a ( $9 \mathrm{kDa}$ ) has remained bound to the alpha'-67.5 kDa fragment. 
A
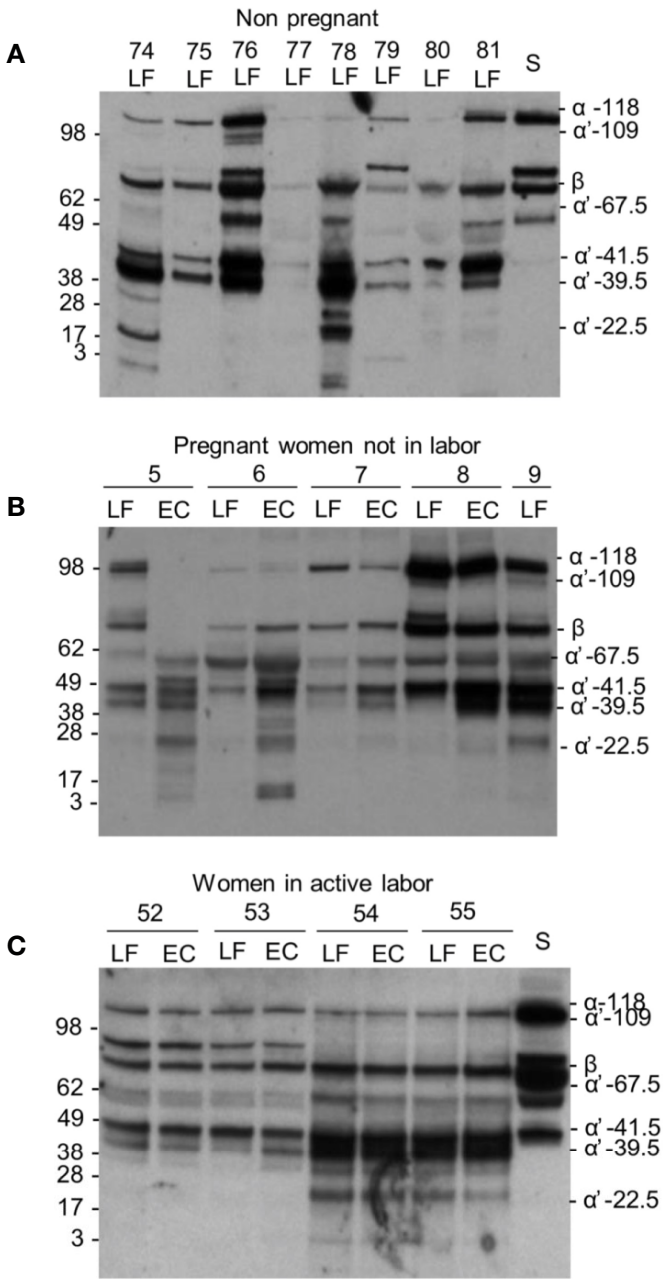

FIGURE 3 | C3 cleavage in the cervicovaginal area. Examples from the three different sample groups are shown. Samples were from: (A) women in the nonpregnant group; (B) pregnant women, who are not in labor and (C) women in active labor but with intact membranes. The cleavage was studied by running the samples in SDS-PAGE under reducing conditions and using Western blotting with an anti-C3c antibody. Sample numbers and types within each group are indicated on the top of the blot. As shown, a wide variation in the extent of $\mathrm{C} 3$ cleavage occurs in the samples. The $\beta$ chain and the key $\alpha$-chain fragments are indicated on the right. Molecular weight markers $(\mathrm{kDa})$ are indicated on the left. S, serum control.

\section{Complement Activation by IgG}

As possible evidence for complement activation by IgG we detected in some samples by Western blotting high molecular weight bands that stained both for the IgG heavy chain and C3 (Figure 5). These bands could present SDS- and reductionresistant covalent complexes between the IgG heavy chain (50 $\mathrm{kDa}$ ) and $\mathrm{C} 3$ activation fragments. The C3-IgG complexes were observed in $10 \%$ of the pregnant women, in $33 \%$ of women at delivery and in $12 \%$ of control women. These complexes could indicate local complex formation between IgG and C3b and thus C3 activation by the IgG antibodies. No similar bands were detected for IgA.

\section{DISCUSSION}

We observed robust local complement activation in the cervicovaginal milieu. C3 activation was seen in women in all the three different groups: when not pregnant, during pregnancy and during labor. High amounts of $\mathrm{C} 3$ cleavage products suggest that the complement system at this site is under continuous state of activation. During labor the complement C3 amounts in samples from the cervicovaginal space appeared as the highest, but the activation percentages were the lowest. IgG was more abundantly present in the reproductive secretions during pregnancy and labor in comparison to the nonpregnant state. In contrast, IgA levels did not differ between the study groups.

Unlike usually assumed for mucosal surfaces, IgG is detected in the cervicovaginal area in addition to IgA (35). We observed higher levels of IgG than IgA in samples from women, who were pregnant or in labor. The opposite, higher levels of mucosal IgA than IgG, was true for nonpregnant women. In body fluids containing IgG and complement, a potential for immune complex formation and complement activation exists. IgG and generation of immune complexes are needed for antibacterial and antiviral defense (36). When comparing the Western blots of $\mathrm{C} 3$ activation fragments and IgG from the same samples, in some samples, similar high molecular weight bands $(>100 \mathrm{kDa})$ were observed (Figure 5) suggesting the presence of covalent complexes between the IgG heavy chain and one or more C3 fragments that remain covalently bound on structures that activated complement. The C3-IgG complexes were more commonly present in samples taken during pregnancy (33\%) than in the other samples (10\% and 12\%). This is a further indication that local complement activation has taken place. It also suggests that local IgG-containing immune complexes could be one of the complement-activating factors. Apart from the obvious factors from the local microbial flora, the nature of local antigens and the relevance of the immune complexes during pregnancy and delivery are unknown. No similar bands were observed for IgA, which is compatible with the fact that IgA does not activate complement.

In recent years, understanding the role of sterile inflammation during pregnancy and labor has increased significantly. Although the complement system has traditionally and evolutionarily been known as part of the host defense against microbes and in causing inflammation, $\mathrm{C}$ also maintains homeostasis by controlling the elimination nonviable tissue components (37-39). Previous studies have suggested that the complement system is involved in pregnancy and parturition, participating in tissue remodeling, recognizing injured cells and enhancing phagocytosis $(10,21,40)$. C3 is abundantly produced by the uterus, and levels of $\mathrm{C} 3$ increase in blood towards the end of pregnancy (26). Also, additional synthesis may occur in the local mucosa (41). Higher amounts, but a lower level, of C3 activation in the cervicovaginal interphase during labor may be related to the dilation and effacement of the cervix, enabling migration of $\mathrm{C} 3$ from plasma. On the basis of our results we cannot say to which extent $\mathrm{C} 3$ is locally produced and how much is diffusing from blood. This likely depends on the situation (e.g. more C3 from blood during delivery) and varies from patient to 


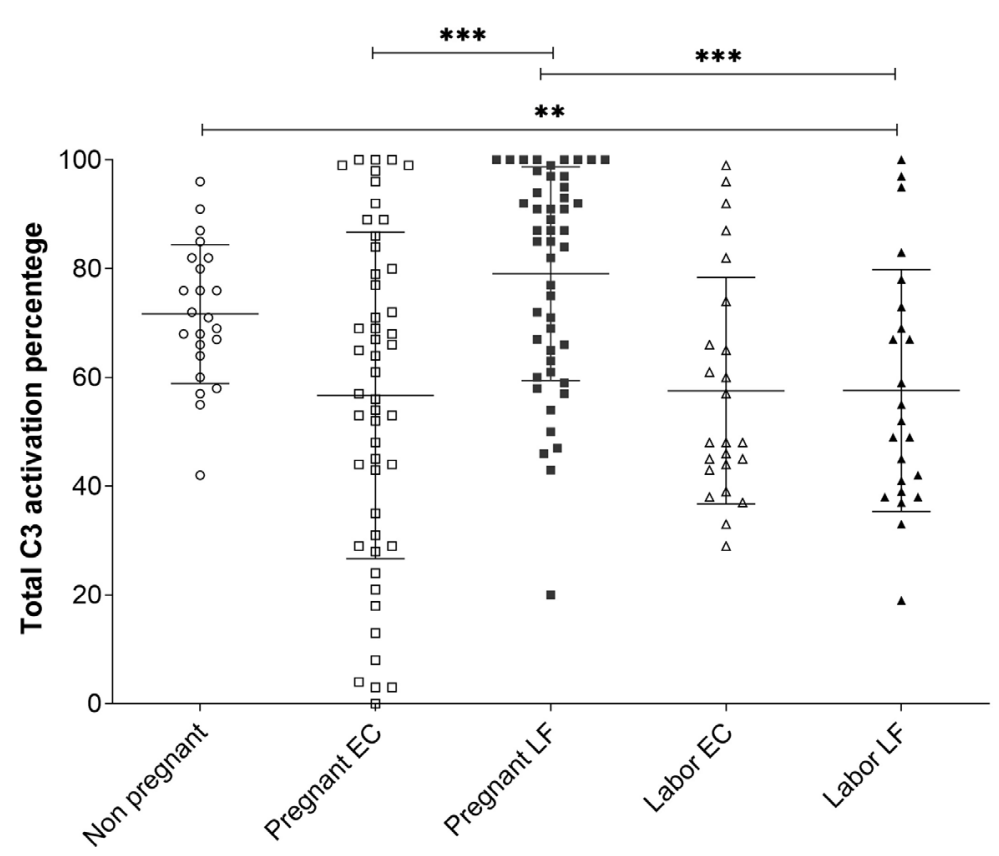

FIGURE 4 | Comparison of C3 activation levels in samples from the EC and the LF in the 3 different groups. The percentages of C3 activation in the three groups were determined from the Western blot analysis of C3 split products using the "ImageJ" program. The mean \pm SD values for the different groups were: nonpregnant women $72 \pm 13 \%$ ( $n=23$ ), pregnant women EC 57 $\pm 30 \%$, LF $79 \pm 20 \%$ ( $n=49$ ) and women in labor $58 \pm 22 \%$ ( $n=24)$ in both the EC and LF. The differences were significant between women at labor vs. pregnant women (***, $\mathrm{P}<0.001$; for LF samples) or nonpregnant women (**, P<0.01) (Tukey's Multiple Comparison Test). The C3 activation percentages in samples taken during pregnancy were significantly higher in the LF $(79 \pm 20 \%$; $n=49)$ than in the $E C$ ( $57 \pm 30 \%$; $n=49)$; $(* \star \star P<$ 0.001). No difference between LF and EC in samples taken at labor were seen.

C3

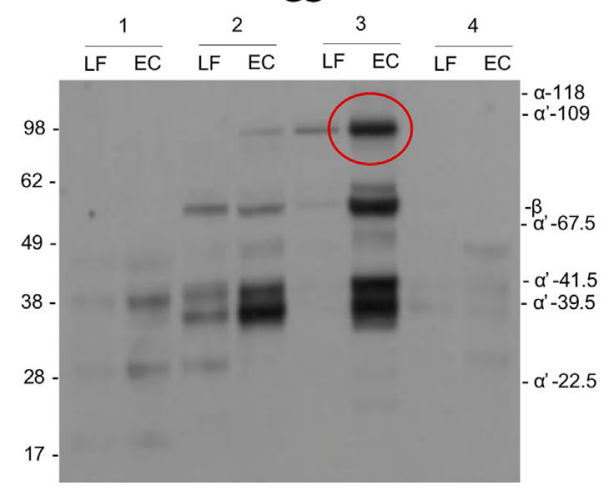

IgG

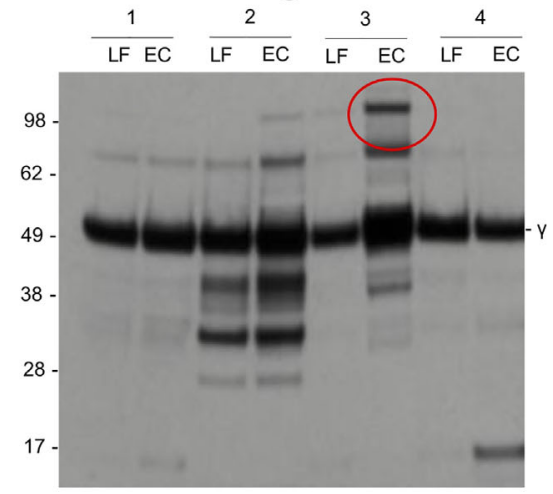

FIGURE 5 | Complement activation by lgG. As evidence for complement activation by lgG we detected in some samples by Western blotting high m.W. bands (circled) that stained both for IgG heavy chain and C3. These bands may present SDS- and reduction-resistant covalent complexes between the IgG heavy chain (50 $\mathrm{kDa}$ ) and a C3 activation fragment. The C3-lgG complexes could indicate local complex formation between lgG and C3b and thus C3 activation by the lgG antibodies.

patient. Blood-contaminated samples (visible red color) were excluded from the analyses. By measuring absorbances at $539 \mathrm{~nm}$ we observed that the highest level of blood contamination in the tested samples was $3.8 \%$. Thus, not all C3 in the samples can originate from contaminating blood.
Our results indicated that complement activation continued to some extent in the LF samples ex vivo, because a difference was seen between samples taken to EDTA or buffer only. In both situations (presence or absence of EDTA) the C3 activation level was higher in the LF samples. A similar difference (stronger 
activation in LF samples; section Local C3 Activation in NonPregnant and Pregnant Women and During Labor) was seen in pregnant women, who were not in labor, but not in women at labor. Thus, although we observed some additional C3 activation in LF samples ex vivo, it did not influence the general conclusions of the study.

The higher activation percentage of $\mathrm{C} 3$ in the LF during pregnancy could result from differences in mucosal secretions and microbiome environments that may affect C3 activation. The lack of difference in C3 activation between the two sites during labor might be explained by the changed anatomical and physiological conditions, like in the fluid and blood inflow during cervical effacement. The lower level of C3 activation during labor suggests that the $\mathrm{C}$ system is in this situation better controlled than other times at this local site. This could also explain the lack of difference between EC and LF samples during labor. Whether the better control of $\mathrm{C}$ activation in the cervicovaginal space during delivery is due to greater amounts of complement regulating proteins, possibly diffusing from circulation, remains to be studied.

Knowledge about the level of local activation and regulation of the complement system during the physiological inflammation process related to delivery is scarce. In general, the activation level of C3 in the cervicovaginal samples was exceptionally high. As examples, an earlier study on local complement activation in otitis media in children reported an up to $40 \%$ level of C3 activation (34) and in dense deposit disease a nearly 100\% level of C3 activation in blood plasma could be reached (42). Like in the middle ear space in otitis media, also in vagina many potential factors could contribute to $\mathrm{C}$ activation and $\mathrm{C} 3$ cleavage. They include microbes, sperm cells, damaged host cells, migrating leukocytes and proteolytic enzymes, like plasmin or matrix metalloproteinases. The cervicovaginal area is thus a true interphase, where a homeostatic balance must persist. The lower genital tract epithelium consists of multiple cell layers of stratified squamous epithelial cells that lack tight junctions. Therefore, it can allow the movement of small molecules, including complement components, through the cell layers. The high level of estrogen at the end of pregnancy increases estradiol receptor expression in cells of the reproductive tract (epithelial cells, macrophages, stromal cells, and lymphocytes). This affects the lower genital tract immunity and promotes complement C3 synthesis (43). What we observed, could thus partially be due to locally produced C3. While complement activation likely has a beneficial function, it may also be harmful. Maternal systemic complement overactivity may result in fetal damage, coagulation disorders, or excessive bleeding during delivery and postpartum. Therefore, complement needs to be carefully controlled. Complement activation can be regulated by local inhibitors or by those coming from circulation. The regulation of complement in this local environment merits more studies.

Quantification of IgG suggested that like for C3, IgG was more abundant in the samples from women in labor than in the other groups. Previous studies suggest that most mucosal IgG originates from blood plasma (7). Our results suggest that IgG is naturally present in the cervicovaginal mucosa. Part of it could derive from the local immune tissue. We have previously demonstrated the presence and activation of local lymphoid tissue including B cells in the vulvovaginal area (44). The presence and activities of IgG in promoting both complement activation and opsonophagocytosis can protect the cervicovaginal space from infections. However, IgG may also make this region vulnerable to inflammation, e.g. by activation of complement and leukocytes, which can generate reactive oxygen metabolites and other mediators of inflammation. Apparently, the balance between these two phenomena varies according to the different physiological challenges, including pregnancy and delivery. It may also depend on the expression of cellular receptors for both activated complement components, like CR1, CR3, C5aR type 1 and for IgG, i.e. the Fc receptors. The different types of Fc receptors can bind different subclasses of IgG and be linked either to activating or inhibiting submembranous domains. The net effects of receptor interactions could thus range from proinflammatory activation to more homeostatic clearance responses. During and after delivery, the need for repair processes is naturally very high. Activation of C3 to C3b (for CR1), and especially for $\mathrm{iC} 3 \mathrm{~b}$ (for CR3) thus probably plays an essential role in promoting clearance of damaged tissue components and healing at the end. In addition, antigen-bound C3dg and C3d could, via their receptor CR2 (CD21), favor activation of B cells and antibody synthesis in the local immune tissue. C5a in turn would promote inflammation through its phlogistic activities via C5aR1.

Hereby we have demonstrated a key activity, activation of complement C3, in the local cervicovaginal immune system. What are the beneficial functions and what are the potential risks need to be analyzed in further studies. Also, the present study did not address the complement inhibitors, which could operate to avoid a full-blown complement activation before the baby enters the birth canal. The presence of local, mucosal IgG implies that this area is an immunologically intermediate region, where both mucosal IgA-based immunity and parenteral IgG-based active immunity coexist. Both may be needed for an active, yet not too excessive inflammatory immune response against potential pathogens. Whether there also is a more direct effect on successful pregnancy or delivery remains an important topic for further studies.

The main strength of the study is the unique sample material obtained from volunteers. The samples were precisely timed, localized and in all cases taken by two experienced doctors. Pregnant women were sampled in the "late term" stage, during which, for unknown reasons, the labor had not yet started. Whether delayed delivery is related in any way to complement dysfunction is not known. Multiple activities of complement in inflammation, in regulating blood flow, cell migration, and activation suggest that complement could have a role in the induction of labor.

In conclusion, our study demonstrates a robust local activation of the complement system and the presence of $\operatorname{IgG}$, in addition to IgA, in the cervicovaginal interface both under normal circumstances, during pregnancy and during delivery. This indicates the presence of an active humoral immune system in this important area. The physiological and potential 
pathophysiological consequences of this phenomenon remain to be worked out in further studies.

\section{DATA AVAILABILITY STATEMENT}

The original contributions presented in the study are included in the article/supplementary material. Further inquiries can be directed to the corresponding authors.

\section{ETHICS STATEMENT}

The studies involving human participants were reviewed and approved by Helsinki University Hospital's Ethical Committee (91/13/03/03/2015). The patients/participants provided their written informed consent to participate in this study.

\section{AUTHOR CONTRIBUTIONS}

SL and JH-E collected samples and. performed the measurements, $\mathrm{HJ}$, IL, and IK were involved in planning and supervised the work,

\section{REFERENCES}

1. Hunt JS. Stranger in a strange land. Immunol Rev (2006) 213:36-47. doi: 10.1111/j.1600-065X.2006.00436.x

2. Robertson SA. Immune regulation of conception and embryo implantationall about quality control? J Reprod Immunol (2010) 85(1):51-7. doi: 10.1016/ j.jri.2010.01.008

3. Stoller M, Traupe T, Khattab AA, de Marchi SF, Steck H, Seiler C. Effects of coronary sinus occlusion on myocardial ischaemia in humans: role of coronary collateral function. Heart (2013) 99(8):548-55. doi: 10.1136/ heartjnl-2012-303305

4. Wang YY, Kannan A, Nunn KL, Murphy MA, Subramani DB, Moench T, et al. IgG in cervicovaginal mucus traps HSV and prevents vaginal herpes infections. Mucosal Immunol (2014) 7(5):1036-44. doi: 10.1038/ mi.2013.120

5. Bard E, Riethmuller D, Biichle S, Meillet D, Pretet JL, Mougin C, et al. Validation of a high sensitive immunoenzymatic assay to establish the origin of immunoglobulins in female genital secretions. J Immunoassay Immunochem (2002) 23(2):145-62. doi: 10.1081/IAS-120003658

6. Tommola P, Butzow R, Unkila-Kallio L, Paavonen J, Meri S. Activation of vestibule-associated lymphoid tissue in localized provoked vulvodynia. Am J Obstet Gynecol (2015) 212(4):476 e1-8. doi: 10.1016/j.ajog.2014.10.1098

7. Thomson AJ, Telfer JF, Young A, Campbell S, Stewart CJ, Cameron IT, et al. Leukocytes infiltrate the myometrium during human parturition: further evidence that labour is an inflammatory process. Hum Reprod (Oxford England) (1999) 14(1):229-36. doi: 10.1093/humrep/14.1.229

8. Gomez-Lopez N, Tanaka S, Zaeem Z, Metz GA, Olson DM. Maternal circulating leukocytes display early chemotactic responsiveness during late gestation. BMC Pregnancy Childbirth (2013) 13 Suppl 1:S8. doi: 10.1186/ 1471-2393-13-S1-S8

9. Young A, Thomson AJ, Ledingham M, Jordan F, Greer IA, Norman JE. Immunolocalization of proinflammatory cytokines in myometrium, cervix, and fetal membranes during human parturition at term. Biol Reproduction (2002) 66(2):445-9. doi: 10.1095/biolreprod66.2.445

10. Girardi G. Complement activation, a threat to pregnancy. Semin Immunopathol (2018) 40(1):103-11. doi: 10.1007/s00281-017-0645-x
SL, HJ, IK, and SM processed the experimental data, performed the analysis, drafted the manuscript and designed the figures. SL, HJ, IK, IL PN, and SM aided in interpreting the results and worked on the manuscript. All authors contributed to the article and approved the submitted version.

\section{FUNDING}

The study was supported by the the Sigrid Jusélius Foundation (4708373 to SM, P52483 to IK), the Academy of Finland (292393 to SM, 324944 to IK) and State Funding to the Helsinki University Hospitals (TYH, VTR): TYH2017110 (PN), TYH2020401 (IK) and TYH2018313, TYH2019311 (SM).

\section{ACKNOWLEDGMENTS}

The authors thank all women, who participated in the study. Juha Kotimaa, Marcel Messing, and Marjo Rissanen are thanked for technical assistance. A special thanks for Rovers Medical Devices, Oss, Netherlands, for donating the sampling brush devices.

11. Nadeau-Vallee M, Obari D, Palacios J, Brien ME, Duval C, Chemtob S, et al. Sterile inflammation and pregnancy complications: a review. Reproduction (2016) 152(6):R277-R92. doi: 10.1530/REP-16-0453

12. Romero R, Xu Y, Plazyo O, Chaemsaithong P, Chaiworapongsa T, Unkel R, et al. A Role for the Inflammasome in Spontaneous Labor at Term. Am J Reprod Immunol (2018) 79:e12440. doi: 10.1111/aji.12440

13. Noris M, Remuzzi G. Overview of complement activation and regulation. Semin Nephrol (2013) 33(6):479-92. doi: 10.1016/j.semnephrol.2013.08.001

14. Thurman JM, Holers VM. The central role of the alternative complement pathway in human disease. J Immunol (2006) 176(3):1305-10. doi: 10.4049/ jimmunol.176.3.1305

15. Botto M, Kirschfink M, Macor P, Pickering MC, Wurzner R, Tedesco F. Complement in human diseases: Lessons from complement deficiencies. $\mathrm{Mol}$ Immunol (2009) 46(14):2774-83. doi: 10.1016/j.molimm.2009.04.029

16. Walport MJ. Complement. Second of two parts. N Engl J Med (2001) 344 (15):1140-4. doi: 10.1056/NEJM200104123441506

17. Ricklin D, Hajishengallis G, Yang K, Lambris JD. Complement: a key system for immune surveillance and homeostasis. Nat Immunol (2010) 11(9):785-97. doi: $10.1038 /$ ni. 1923

18. Baines M, Jarvis A, Nisbet JA. Adaption of SMA 12-60 methodology to the analysis of urine. Clinica chimica acta; Int J Clin Chem (1974) 53(3):331-8. doi: 10.1016/0009-8981(74)90272-1

19. Richani K, Soto E, Romero R, Espinoza J, Chaiworapongsa T, Nien JK, et al. Normal pregnancy is characterized by systemic activation of the complement system. J Maternal Fetal Neonatal Med Off J Eur Assoc Perinatal Medicine Fed Asia Oceania Perinatal Societies Int Soc Perinatal Obstet (2005) 17(4):239-45. doi: 10.1080/14767050500072722

20. Segura-Cervantes E, Mancilla-Ramirez J, Zurita L, Paredes Y, Arredondo JL, Galindo-Sevilla N. Blood SC5b-9 complement levels increase at parturition during term and preterm labor. J Reprod Immunol (2015) 109:24-30. doi: 10.1016/j.jri.2015.02.008

21. Girardi G, Bulla R, Salmon JE, Tedesco F. The complement system in the pathophysiology of pregnancy. Mol Immunol (2006) 43(1-2):68-77. doi: 10.1016/j.molimm.2005.06.017

22. Kovar IZ, Riches PG. C3 and C4 complement components and acute phase proteins in late pregnancy and parturition. J Clin Pathol (1988) 41(6):650-2. doi: $10.1136 /$ jcp.41.6.650 
23. Girardi G, Salmon JB. The role of complement in pregnancy and fetal loss. Autoimmunity (2003) 36(1):19-26. doi: 10.1080/0891693031000067322

24. Regal JF, Gilbert JS, Burwick RM. The complement system and adverse pregnancy outcomes. Mol Immunol (2015) 67(1):56-70. doi: 10.1016/ j.molimm.2015.02.030

25. Teisner B, Hau J, Tucker M, Lahood J, Grudzinskas JG. Circulating C3, C4, and C3 split products (C3c and C3d) during normal pregnancy. Am J Reprod Immunol (1982) 2(6):309-11. doi: 10.1111/j.1600-0897.1982.tb00198.x

26. He YD, Xu BN, Song D, Wang YQ, Yu F, Chen Q, et al. Normal range of complement components during pregnancy: A prospective study. Am J Reprod Immunol (2020) 83(2):e13202. doi: 10.1111/aji.13202

27. Lokki AI, Heikkinen-Eloranta J, Jarva H, Saisto T, Lokki ML, Laivuori H, et al. Complement activation and regulation in preeclamptic placenta. Front Immunol (2014) 5:312. doi: 10.3389/fimmu.2014.00312

28. Lokki AI, Kaartokallio T, Holmberg V, Onkamo P, Koskinen LLE, Saavalainen P, et al. Analysis of Complement C3 Gene Reveals Susceptibility to Severe Preeclampsia. Front Immunol (2017) 8:589. doi: 10.3389/fimmu.2017.00589

29. Breen KA, Seed P, Parmar K, Moore GW, Stuart-Smith SE, Hunt BJ. Complement activation in patients with isolated antiphospholipid antibodies or primary antiphospholipid syndrome. Thromb Haemostasis (2012) 107(3):423-9. doi: 10.1160/TH11-08-0554

30. Lynch AM, Eckel RH, Murphy JR, Gibbs RS, West NA, Giclas PC, et al. Prepregnancy obesity and complement system activation in early pregnancy and the subsequent development of preeclampsia. Am J Obstet Gynecol (2012) 206(5):428.e1-8. doi: 10.1016/j.ajog.2012.02.035

31. Lynch AM, Gibbs RS, Murphy JR, Giclas PC, Salmon JE, Holers VM. Early elevations of the complement activation fragment $\mathrm{C} 3 \mathrm{a}$ and adverse pregnancy outcomes. Obstet Gynecol (2011) 117(1):75-83. doi: 10.1097/AOG.0b013 e3181fc3afa

32. Lynch AM, Wagner BD, Deterding RR, Giclas PC, Gibbs RS, Janoff EN, et al. The relationship of circulating proteins in early pregnancy with preterm birth. Am J Obstet Gynecol (2016) 214(4):517 e1-8. doi: 10.1016/j.ajog.2015.11.001

33. Gonzalez JM, Romero R, Girardi G. Comparison of the mechanisms responsible for cervical remodeling in preterm and term labor. $J$ Reprod Immunol (2013) 97(1):112-9. doi: 10.1016/j.jri.2012.07.008

34. Närkiö-Mäkelä M, Teppo AM, Meri S. Complement C3 cleavage and cytokines interleukin-1beta and tumor necrosis factor-alpha in otitis media with effusion. Laryngoscope (2000) 110(10 Pt 1):1745-9. doi: 10.1097/ 00005537-200010000-00035

35. Horton RE, Vidarsson G. Antibodies and their receptors: different potential roles in mucosal defense. Front Immunol (2013) 4:200. doi: 10.3389/ fimmu. 2013.00200
36. Merle NS, Church SE, Fremeaux-Bacchi V, Roumenina LT. Complement System Part I - Molecular Mechanisms of Activation and Regulation. Front Immunol (2015) 6:262. doi: 10.3389/fimmu.2015.00262

37. Dantas E, Diaz FE, Gerber PP, Merlotti A, Varese A, Ostrowski M, et al. Low $\mathrm{pH}$ impairs complement-dependent cytotoxicity against IgG-coated target cells. Oncotarget (2016) 7(45):74203-16. doi: 10.18632/oncotarget.12412

38. Merle NS, Noe R, Halbwachs-Mecarelli L, Fremeaux-Bacchi V, Roumenina LT. Complement System Part II: Role in Immunity. Front Immunol (2015) 6:257. doi: 10.3389/fimmu.2015.00257

39. Teirilä L, Heikkinen-Eloranta J, Kotimaa J, Meri S, Lokki AI. Regulation of the complement system and immunological tolerance in pregnancy. Semin Immunol (2019) 45:101337. doi: 10.1016/j.smim.2019.101337

40. Mollnes TE, Jokiranta TS, Truedsson L, Nilsson B, Rodriguez de Cordoba S, Kirschfink M. Complement analysis in the 21st century. Mol Immunol (2007) 44(16):3838-49. doi: 10.1016/j.molimm.2007.06.150

41. Pudney J, Quayle AJ, Anderson DJ. Immunological microenvironments in the human vagina and cervix: mediators of cellular immunity are concentrated in the cervical transformation zone. Biol Reproduction (2005) 73(6):1253-63. doi: 10.1095/biolreprod.105.043133

42. Meri S, Koistinen V, Miettinen A, Törnroth T, Seppälä IJ. Activation of the alternative pathway of complement by monoclonal lambda light chains in membranoproliferative glomerulonephritis. J Exp Medicine (1992) 175 (4):939-50. doi: 10.1084/jem.175.4.939

43. Brotman RM, Ravel J, Bavoil PM, Gravitt PE, Ghanem KG. Microbiome, sex hormones, and immune responses in the reproductive tract: challenges for vaccine development against sexually transmitted infections. Vaccine (2014) 32(14):1543-52. doi: 10.1016/j.vaccine.2013.10.010

44. Tommola P, Bützow R, Unkila-Kallio L, Paavonen J, Meri S. Activation of vestibule-associated lymphoid tissue in localized provoked vulvodynia. Am J Obstet Gynecol (2015) 212(4):476.e1-8. doi: 10.1016/j.ajog.2014.10.1098

Conflict of Interest: The authors declare that the research was conducted in the absence of any commercial or financial relationships that could be construed as a potential conflict of interest.

Copyright $(2021$ Livson, Jarva, Kalliala, Lokki, Heikkinen-Eloranta, Nieminen and Meri. This is an open-access article distributed under the terms of the Creative Commons Attribution License (CC BY). The use, distribution or reproduction in other forums is permitted, provided the original author(s) and the copyright owner(s) are credited and that the original publication in this journal is cited, in accordance with accepted academic practice. No use, distribution or reproduction is permitted which does not comply with these terms. 